\title{
The Cube of Every Connected Graph is 1-Hamiltonian *
}

\author{
Gary Chartrand and S. F. Kapoor**
}

\begin{abstract}
(November 15, 1968)
Let $G$ be any connected graph on 4 or more points. The graph $G^{3}$ has as its point set that of $G$, and two distinct points $u$ and $v$ are adjacent in $G^{3}$ if and only if the distance between $u$ and $v$ in $G$ is at most three. It is shown that not only is $G^{3}$ hamiltonian, but the removal of any point from $G^{3}$ still yields a hamiltonian graph.
\end{abstract}

Key Words: Cube of a graph; graph; hamiltonian.

Let $G$ be a graph (finite, undirected, with no loops or multiple lines). A walk of $G$ is a finite alternating sequence of points and lines of $G$, beginning and ending with a point and where each line is incident with the points immediately preceding and following it. A walk in which no point is repeated is called a path; the length of a path is the number of lines in it.

A graph $G$ is connected if between every pair of distinct points there exists a path, and for such a graph, the distance between two points $u$ and $v$ is defined as the length of the shortest path if $u \neq v$ and zero if $u=v$.

A walk with at least three points in which the first and last points are the same but all other points are distinct is called a cycle. A cycle containing all points of a graph $G$ is called a hamiltonian cycle of $G$, and $G$ itself a hamiltonian graph.

Throughout the literature of graph theory there have been defined many graph-valued functions $f$ on the class of graphs. In certain instances results have been obtained to show that if $G$ is connected and has sufficiently many points, then the graph $f(G)$ (or its iterates $f^{n}(G)$ ) is a hamiltonian graph. Examples of such include the line-graph function $L(G)$ and the total graph function $T(G)$ (see $[2,1],{ }^{1}$ respectively).

The line-graph $L(G)$ of graph $G$ is a graph whose point set can be put in one-to-one correspondence with the line set of $G$ such that adjacency is preserved. The total graph $T(G)$ has its point set in one-to-one correspondence with the set of points and lines of $G$ in such a way that two points of $T(G)$ are adjacent if and only if the corresponding elements of $G$ are adjacent or incident.

Another example which always yields a hamiltonian graph is the cube function. In fact, if $x$ is any line in a connected graph $G$ with at least three points, then the cube of $G$ has a hamiltonian cycle containing $x$. This follows from a result due to Karaganis [4] by which the cube of any connected graph $G$ on $p(\geqslant 3)$ points turns out to be hamiltonian-connected, i.e., between any two points there exists a path containing all points of $G$. Now if $x$ is any line joining points $u$ and $v$ in $G$, then the addition of $x$ to the hamiltonian path between $u$ and $v$ in the cube of the graph produces a hamiltonian cycle of $G$ containing $x$.

The cube $G^{3}$ of a connected graph $G$ has as its point set that of $G$, and two distinct points $u$ and $v$ are adjacent in $G^{3}$ if and only if the distance between $u$ and $v$ in $G$ is at most three. The purpose of this note is to prove that if $G$ is a connected graph (with $p \geqslant 4$ points) then not only is $G^{3}$ hamiltonian, but the removal of any point from $G^{3}$ still yields a hamiltonian graph. Graphs enjoying this property have been referred to as 1-hamiltonian in [3].

THEOREM. If $\mathrm{G}$ is a connected graph on $\mathrm{p} \geqslant 4$ points, then $\mathrm{G}^{3}$ is 1-hamiltonian.

\footnotetext{
*An invited paper.

**Present address: Western Michigan University, Kalamazoo, Mich. 49001.

${ }^{\prime}$ Figures in brackets indicate the literature references at the end of this paper.
} 
Proof. That $G^{3}$ is hamiltonian is already known; indeed, there exists a hamiltonian cycle of $G^{3}$ which contains any specified line of $G$ as we have already noted. We now show that the deletion of any point from $G^{3}$ also results in a hamiltonian graph. The proof is by induction on $p$, the result being true for $p=4$, since the cube of any connected graph on 4 points yields the complete graph, and the removal of any point from this leaves a cycle on three points. Clearly the theorem follows if the result is proved for a spanning tree $T$ of $G$. Assume the statement to be true for all trees on $n$ points, $4 \leqslant n<p$, and let $T$ be any tree on $p$ points. Consider the forest $F$ obtained by deleting any point $u$ from $T$. Denote the components of $F$ by $T_{i}, 1 \leqslant i \leqslant k$, where $k$ is the degree of $u$ in $T$. Furthermore, let $T_{i}$ have $p_{i}$ points so that $p=1+\sum_{i=1}^{k} p_{i}$, and let $u_{i}$ be the point in $T_{i}$ which is adjacent to
$u$ in $T$.

If $k=1$, then $F$ is a tree. If $p_{1} \geqslant 4$, then $T_{1}^{3}$ is 1 -hamiltonian by hypothesis, while if $p_{1}=3, T_{1}^{3}$ is a triangle; in either case $T_{1}^{3}$ is hamiltonian.

Assume $k>1$. For all $i$ such that $1 \leqslant i \leqslant k$ and $p_{i} \geqslant 3$, it follows from earlier remarks that a hamiltonian cycle $C_{i}$ may be selected in $T_{1}^{3}$ to contain the line $u_{i} v_{i}$ where $v_{i}$ is adjacent to $u_{i}$ in $T_{i}$. If $p_{i}=2, T_{i}$ has only two points, namely $u_{i}$ and $v_{i}$. We now construct a hamiltonian cycle $C$ in $T^{3}-u$ as follows.

For each $C_{i}$ so defined for $p_{i} \geqslant 3$, remove the line $u_{i} v_{i}$ to obtain a path $P_{i}$ beginning at $u_{i}$ and ending at $v_{i}$. If $p_{i}=2$, then $T_{i}$ is itself a path $P_{i}$ beginning at $u_{i}$ and ending at $v_{i}$. For completeness, if $p_{i}=1$, we assign a second label $v_{i}$ to the point $u_{i}$ and speak of the trivial path $P_{i}$. Observe that for $r \neq s$, the distance from $v_{r}$ to $u_{s}$ is two if $P_{r}$ is trivial, and is three otherwise. In either case the line $v_{r} u_{s}$ is present in $T^{3}-u$. The desired cycle $C$ is $P_{1}, v_{1} u_{2}, P_{2}, \ldots, P_{j}, v_{j} u_{j+1}, P_{j+1}, \ldots, P_{k}, v_{k} u_{1}$.

The previous result cannot be improved so that the removal of any two points from the cube of a connected graph with at least five points results in a hamiltonian graph. For example, if $P$ is a path containing adjacent points $u$ and $v$, neither an end-point, then $P^{3}-\{u, v\}$ is not hamiltonian.

\section{References}

[1] Behzad, M., Chartrand, G., Total graphs and traversability, Proc. Edinburgh Math. Soc. 15, 117-120 (1966).

[2] Chartrand, G., The existence of complete cycles in repeated line-graphs, Bull. Amer. Math. Soc. 71, 668-670 (1965).

[3] Chartrand, G., Kapoor, S. F., and Lick, D. R., n-Hamiltonian graphs, J. Combinatorial Theory, to appear.

[4] Karaganis, J. J., On the cube of a graph, Canad. Math. Bull. 11, 295-296 (1968).

(Paper 73B1-287) 